\title{
Lowering the background level and the energy threshold of Micromegas x-ray detectors for axion searches
}

F.J. Iguaz*, F. Aznar ; J.F. Castel, T. Dafni, J.A. Garcia, J.G. Garza, I.G. Irastorza, I. Ortega; A. Rodríguez and A. Tomas ${ }^{\S}$

Laboratorio de Física Nuclear y Astropartículas, Universidad de Zaragoza, Spain.

E-mail: iguaz@cern.ch

\section{S. Aune, E. Ferrer-Ribas, J. Galan, I. Giomataris, D. Jourde and T. Papaevangelou}

Service d'Electronique, des Détecteurs et d'Informatique, CEA-Saclay, Gif-sur-Yvette, France

\section{Davenport and T. Vafeiadis}

CERN, European Organization for Particle Physics and Nuclear Research, Geneva, Switzerland

\section{S.C. Yildiz}

Department of Physics, Dogus University, Acibadem Kadikoy, Istanbul, Turkey

Axion helioscopes search for solar axions by their conversion in x-rays in the presence of high magnetic fields. The use of low background x-ray detectors is an essential component contributing to the sensitivity of these searches. In this work, we review the recent advances on Micromegas detectors used in the CERN Axion Solar Telescope (CAST) and proposed for the future International Axion Observatory (IAXO). The actual setup in CAST has achieved background levels below $10^{-6} \mathrm{keV}^{-1} \mathrm{~cm}^{-2} \mathrm{~s}^{-1}$, a factor 100 lower than the first generation of Micromegas detectors. This reduction is based on active and passive shielding techniques, the selection of radiopure materials, offline discrimination techniques and the high granularity of the readout. We describe in detail the background model of the detector, based on its operation at CAST site and at the Canfranc Underground Laboratory (LSC), as well as on Geant4 simulations. The best levels currently achieved at LSC are low than $10^{-7} \mathrm{keV}^{-1} \mathrm{~cm}^{-2} \mathrm{~s}^{-1}$ and show good prospects for the application of this technology in IAXO. Finally, we present some ideas and results for reducing the energy threshold of these detectors below $1 \mathrm{keV}$, using high-transparent windows, autotrigger electronics and studying the cluster shape at different energies. As a high flux of axion-like-particles is expected in this energy range, a sub-keV threshold detector could enlarge the physics case of axion helioscopes.

Technology and Instrumentation in Particle Physics 2014

2-6 June, 2014

Amsterdam, the Netherlands

\footnotetext{
* Speaker.

${ }^{\dagger}$ Present address: Centro Universitario de la Defensa, Universidad de Zaragoza, Zaragoza, Spain

*Present address: CERN, European Organization for Particle Physics and Nuclear Research.

${ }^{\S}$ Present address: High Energy Physics group, Blackett Laboratory, Imperial College, London, U.K.
} 


\section{Micromegas for axion searches}

Axion helioscopes [1] aim to detect solar axions through their conversion by the inverse Primakoff effect into x-rays (1-10 keV) using strong magnetic fields. The CERN Axion Solar Telescope (CAST) [2] is the most powerful example and has set the best exclusion limits in the axionphoton coupling for a wide range of axion masses $[3,4]$. Three of the four CAST magnet bores are equipped with microbulk Micromegas (MM) detectors $[5,6]$ as they have a light and radiopure material budget [7]; show an excellent energy resolution (12\% FWHM at $5.9 \mathrm{keV})$ [8]; have a high discrimination power to select x-rays (point-like events) from muons and gammas; can be shielded by applying the same techniques used in rare events experiments.

X-ray detectors are an important issue for the future International Axion Observatory (IAXO) $[9,10]$. Its main goal is to improve the signal-to-noise ratio more than $10^{4}$ with respect to CAST, i.e. 1-2 orders of magnitude in sensitivity to the axion-photon coupling. From the different improvements foreseen, low background $\mathrm{x}$-rays detectors must reach values in $10^{-7}-10^{-8} \mathrm{~s}^{-1} \mathrm{keV}^{-1}$ $\mathrm{cm}^{-2}$. MM technology is a perfect candidate according to the latest level reached in CAST-MM during 2013 [11] and currently the limit achieved at the Canfranc Underground Laboratory (LSC).

In section 2, we will briefly describe the actual CAST-MM background model (exposed in detail in [6]). We will thus present the physical motivation and the R\&D for sub-keV CAST-MM detectors in section $\mathbf{3}$ and, we will finish with some conclusions and prospects.

\section{Background model of CAST-MM detectors}

In table 1 the different contributions to background model of CAST-MM detectors are detailed. They have been identified either by in-situ measurements at CAST, by a detector's replica installed at LSC or by Geant 4 simulations. The main contribution is caused by muons, while the origin of actual limit set at LSC is unknown. Some hypothesis are neutrons or the ${ }^{39} \mathrm{Ar}$ isotope.

\begin{tabular}{c|cc|c} 
Contribution & \multicolumn{2}{|c|}{ Level $\left(\mathrm{s}^{-1} \mathrm{keV}^{-1} \mathrm{~cm}^{-2}\right)$} & Shielding technique applied \\
& Before & After & \\
\hline Gamma flux & $7 \times 10^{-5}$ & None? & Full coverage by $10 \mathrm{~cm}$ lead shielding \\
Radon & $8 \times 10^{-7}$ & None & Nitrogen flux inside the shielding \\
Cosmic muons & $2 \times 10^{-6}$ & $6 \times 10^{-7}$ & $95 \%$ coverage by an active muon veto \\
Al cathode & $5 \times 10^{-7}$ & None & Replacement by an ultrapure copper cathode \\
\hline LSC limit & \multicolumn{2}{|c|}{$1.1 \times 10^{-7}$} & Neutrons? ${ }^{39} \mathrm{Ar}$ Others?
\end{tabular}

Table 1: The different contributions to background level in 2-7 keV range identified in CAST-MM background model and the different shielding techniques applied to completely or partially remove them. For clarity, values are given without errors. The full measurements with the associated errors are in [6].

\section{Lowering the energy threshold}

New calculations of axion production at the Sun by the so-called "BCA processes" in nonhadronic axion models [12] point out to an axion flux peaking at energies around $1 \mathrm{keV}$. This fact motivates the use of sub-keV detectors in IAXO. With this aim, there is a R\&D line based on: 
Window The $\mathrm{x}$-rays coming from the magnet enter the conversion volume via a gas-tight window made of $5 \mu \mathrm{m}$ aluminized mylar foil. This foil is supported by a metallic squared-pattern strong-back, to withstand the pressure difference to the magnet's vacuum system. This foil is transparent down to energies of $1.5 \mathrm{keV}$. Other possible materials like polyimide-based foils are being studied, as their transparency is enlarged down to $0.7 \mathrm{keV}$.

Gas Other mixtures than Ar+2\%isobutane have been studied to increase the actual operation point $\left(8 \times 10^{3}\right)$, i.e, to decrease the energy threshold. A value of $3 \times 10^{4}$ is reached in neon-based mixtures but the pressure must be increased to maximize the photon conversion [8].

Electronics The actual one is based on the AFTER chip [13], which provides time information of each strip. This feature improves the signal-to-noise ratio and have reduced the energy threshold down to $450 \mathrm{eV}$. Autotrigger systems like AGET [14] may further enhance this reduction.

Analysis The CAST-MM detectors have been calibrated in an electron beam at the CAST Detector Laboratory [15]. In this setup, the fluorescence lines of different target materials ranging from 2.3 (gold) to $8.0 \mathrm{keV}$ (copper) are used to calculate the integrated signal efficiency [11]. This data also provides information on event's topology, which can be used in the analysis. To illustrate this issue, the distribution of the cluster's width in Z (left) and the cluster difference between $X \& Y$ widths (right) are shown respectively in figure 1 . Clusters are wider at low energies because most of the X-rays may be absorbed in the first milimeters just after the window and will suffer more diffusion effects. Cluster differences also increase at low energies as charge fluctuations between the two detector planes (XY) are more important.
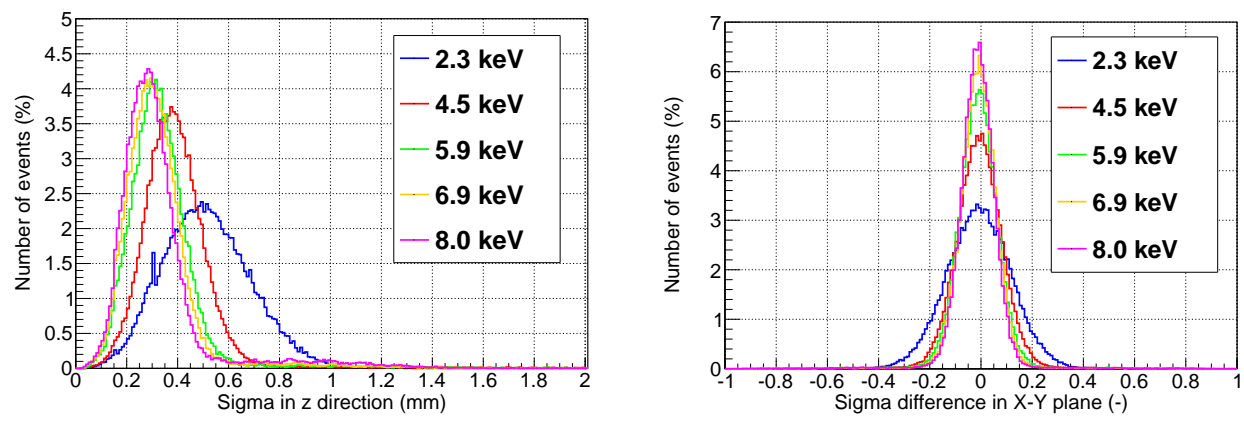

Figure 1: Distribution of the cluster's width in Z (left, in sigma units) and the cluster's width difference in XY (right) for the different fluorescence lines used in the x-ray calibration of CAST-M18.

\section{Conclusions and prospects}

During more than a decade in CAST, Micromegas detectors have reduced their background levels by two orders of magnitude, reaching a value of $7 \times 10^{-7} \mathrm{keV}^{-1} \mathrm{~cm}^{-2} \mathrm{~s}^{-1}$. Most of contributions have been identified and removed applying shielding techniques used in rare event experiments. The best levels currently achieved at LSC (a factor 7 lower) show good prospects for the application of this technology in IAXO. Recent calculations of axion flux motivate the use of sub$\mathrm{keV}$ detectors in CAST. We have presented the main open R\&D lines on this issue for CAST-MM. 


\section{Acknowledgments}

We thank to our colleagues of CAST for many years of collaborative work, R. de Oliveira and his team at CERN for the manufacturing of the microbulk readouts and the LSC staff for their support during the use of their facilities. We acknowledge the support from the European Commission under the European Research Council T-REX Starting Grant ref. ERC-2009-StG-240054 of the IDEAS program of the 7th EU Framework Program. We also the acknowledge support from the Spanish Ministry MINECO under contracts ref. FPA2008-03456 and FPA2011-24058, as well as under the CPAN project ref. CSD2007-00042 from the Consolider-Ingenio 2010 program. These grants are partially funded by the European Regional Development funded (ERDF/FEDER). My personal gratitude is for the support from the Juan de la Cierva program.

\section{References}

[1] T. Dafni and F.J. Iguaz, Axion helioscopes update: the status of CAST and IAXO, in proceedings of TIPP 2014 conference, POS (TIPP 2014) 130.

[2] CAST Collaboration, K. Zioutas et al., First results from the CERN Axion Solar Telescope (CAST), Phys. Rev. Lett. 94 (2005) 121301.

[3] CAST Collaboration, S. Andriamonje et al., An improved limit on the axion-photon coupling from the CAST experiment, JCAP 04 (2007) 010.

[4] E. Arik et al., Search for solar axions by the CERN axion solar telescope with ${ }^{3}$ He buffer gas: closing the hot dark matter gap, Phys. Rev. Lett. 112 (2014) 091302.

[5] S. Andriamonje et al., Development and performance of Microbulk Micromegas detectors, JINST 5 (2010) P02001.

[6] S. Aune et al., Low background $x$-ray detection with Micromegas for axion search, JINST 9 (2014) P01001.

[7] S. Cebrián et al., Radiopurity of micromegas readout planes, Astropart. Phys. 34 (2011) 354.

[8] F.J. Iguaz et al., Characterization of microbulk detectors in argon- and neon-based mixtures, JINST 7 (2012) P04007.

[9] I.G. Irastorza et al., The International Axion Observatory IAXO, Letter of Intent to the CERN SPS commitee, CERN-SPSC-2013-022, SPSC-I-242 (2013).

[10] E. Armengaud et al., Conceptual design of the International Axion Observatory (IAXO), JINST 9 (2014) T05003.

[11] J.G. Garza et al., X-ray detection with Micromegas with background levels below $10^{-6} \mathrm{keV}^{-1} \mathrm{~cm}^{-2}$ $s^{-1}$, JINST 8 (2013) C12042.

[12] J. Redondo, Solar axion-flux from the axion-electron coupling, JCAP 12 (2013) 008.

[13] P. Baron et al., AFTER, an ASIC for the readout of the large T2K time projection chambers, IEEE Trans. Nucl. Sci. 55 (2008) 1744.

[14] S. Anvar et al., AGET, the GET front-end ASIC, for the readout of the time projection chambers used in nuclear physics experiments, IEEE NSS/MIC (2011) 745.

[15] T. Vafeiadis, Contribution to the search for solar axions in the CAST experiment, $\mathrm{PhD}$ thesis, Aristotle University of Thessaloniki, Thessaloniki (Greece), 2013. CERN-THESIS-2012-349. 